Check for updates

Cite this: Phys. Chem. Chem. Phys. 2021, 23, 8900

Received 31st January 2021

Accepted 29th March 2021

DOI: $10.1039 / d 1 c p 00453 k$

rsc.li/pccp

\title{
Excited state dynamics of BODIPY-based acceptor-donor-acceptor systems: a combined experimental and computational study $\dagger$
}

\author{
Zimu Wei, ${ }^{a}$ Sushil Sharma, ${ }^{\mathrm{b}}$ Abbey M. Philip, ${ }^{\mathrm{a}}$ Sanchita Sengupta (D) ${ }^{\mathrm{b}}$ and \\ Ferdinand C. Grozema (D)*a
}

\begin{abstract}
Donor-bridge-acceptor systems based on boron dipyrromethene (BODIPY) are attractive candidates for bio-imagining and sensing applications because of their sensitivity to temperature, micro-viscosity and solvent polarity. The optimization of the properties of such molecular sensors requires a detailed knowledge of the relation between the structure and the photophysical behavior in different environments. In this work we have investigated the excited-state dynamics of three acceptor-donoracceptor molecules based on benzodithiophene and BODIPY in solvents of different polarities using a combination of ultrafast spectroscopy and DFT-based electronic structure calculations. Transient absorption spectra show that upon photoexcitation an initial excited species with an induced absorption band in the near-infrared regime is formed independent of the solvent polarity. The subsequent photophysical processes strongly depend on the solvent polarity. In non-polar toluene this initial excited state undergoes a structural relaxation leading to a delocalized state with partial charge transfer character, while in the more polar tetrahydrofuran a fully charge separated state is formed. The results clearly show how factors such as donor-acceptor distance and restricted rotational motion by steric hindrance can be used to tune the excited state photophysics to optimize such systems for specific applications.
\end{abstract}

\section{Introduction}

Fluorescent molecular rotors that are sensitive to the temperature and micro-viscosity variations have been extensively explored for applications in sensing, ${ }^{1,2}$ bio-imaging $^{3-5}$ and diagnosis. ${ }^{6,7}$ Applications of such molecular sensors can be further extended to detection of metal ions, ${ }^{8,9}$ humidity detection ${ }^{10}$ and optoelectronics, ${ }^{11}$ by incorporation of a donor-acceptor (D-A) system. Upon photoexciting such a D-A system it may either exhibit photoemission from the local excited (LE) state that is formed on photoexcitation, or it may emit at a longer wavelength from a charge transfer (CT) state where electrons and holes are spatially apart from each other. Depending on the equilibrium between the LE state formed upon the excitation and the CT state formed by the subsequent photoinduced charge transfer, the molecules can exhibit dual emission with a large Stokes shift, resulting in a high

\footnotetext{
${ }^{a}$ Department of Chemical Engineering, Delft University of Technology, Delft, The Netherlands. E-mail: f.c.grozema@tudelft.nl

${ }^{b}$ Department of Chemical Sciences, Indian Institute of Science Education and Research (IISER) Mohali, Punjab-140306, India

$\dagger$ Electronic supplementary information (ESI) available. See DOI: 10.1039/ d1cp00453k
}

sensor accuracy ${ }^{12}$ and the emission of white light. ${ }^{13}$ Both the emission probability and the rate of formation of the CT state are directly influenced by subtle variations in the molecular structure, and hence such variations can lead to significant changes in the excited state behavior. Therefore, understanding the underlying photophysical mechanisms governing the charge transfer process is of crucial importance in optimizing the design of such systems.

The rational design of a D-A system entails a proper selection of the electron donating and electron accepting moieties. Benzodithiophene (BDT) is well known for its remarkable performance as a building block in organic photovoltaics. ${ }^{14-16}$ Recently, it has been reported to be a promising candidate as the donor unit in functional dyads and triads. ${ }^{17,18}$ On the other hand, boron dipyrromethene (BODIPY) derivatives have been widely used as fluorophores as well as electron acceptors in fluorescent molecular probes ${ }^{19,20}$ due to their large extinction coefficients, ${ }^{21,22}$ long excited state lifetimes, ${ }^{23}$ high photostability, ${ }^{24}$ various modification possibilities ${ }^{25}$ and their intrinsic electron-accepting ability. However, only a few red emissive BODIPY based D-A rotor molecules with large Stokes shifts have been reported, ${ }^{26-28}$ and their quantitative photophysical characterizations regarding solvent dependence and 

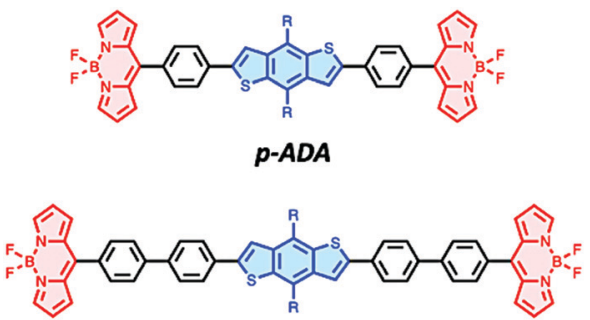

pp-ADA
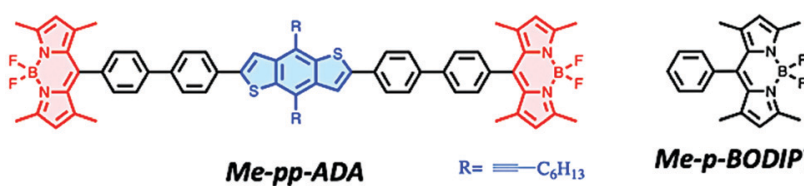

Me-p-BODIPY

Fig. 1 Chemical structures of ADA triads and their model acceptors.

steric restrictions on the ultrafast time scale, to our knowledge, have not been studied.

To gain more insight in the relation between molecular conformation and the photophysics, we have investigated the photophysical properties of a series of acceptor-donor-acceptor triads based on a BDT donor and two BODIPY acceptors (Fig. 1) that are of interest for molecular temperature/viscosity sensors and opto-electronics. In these D-A systems two electron accepting BODIPY units are connected to the BDT donor at the meso positions, with either one or two intervening phenyl groups to investigate the effect of D-A separation on the photophysics. In addition, introducing four methyl groups at the 1-, 3-, 5-, 7-positions in each BODIPY unit allows to study the effect of the mutual rotation angle, and hence the electronic coupling between the donor and acceptor unit without changing the D-A distance. We have used a combination of femtosecond time-resolved transient absorption (TA) spectroscopy and time-dependent density functional theory (TDDFT) calculations. The results show an unanticipated delocalization of the initial excited state in p-ADA and $\mathbf{p p - A D A}$ that can initiate a fast charge transfer process in polar solvents. In addition, we have elucidated the role of the steric hindrance in the photophysical properties of Me-pp-ADA. These results offer new insight in the relation between the photophysics of BODIPY based fluorescent molecular rotor systems that will aid in the optimization of their properties for future applications.

\section{Experimental}

\section{Materials}

For all the spectroscopic measurements, spectroscopy-grade solvents were purchased from commercial suppliers and used as received. The model acceptor Me-p-BODIPY (1,3,5,7tetramethyl-8-phenyl-4,4-difluoroboradiazaindacene) was purchased from Sigma-Aldrich. The model acceptor p-BODIPY was synthesized according to the previous reported procedure $^{29}$ and the characterization is presented in the ESI $\dagger$ (Fig. S10 and S11). The synthesis of p-ADA, pp-ADA and Me-ppADA were published in ref. 30 and 31 .

\section{Optical characterization}

All the spectroscopic measurements were carried out at room temperature. Steady-state absorption spectra were measured by PerkinElmer Lambda UV/Vis spectrophotometer using a quartz cuvette with a $1 \mathrm{~cm}$ path length. Steady-state fluorescence emission spectra were measured using an Edinburgh FLS980 spectrometer excited at $500 \mathrm{~nm}$. Fluorescence lifetimes were recorded using an Edinburgh LifeSpec-ps spectrometer with a fixed excitation wavelength of $404 \mathrm{~nm}$.

\section{Femtosecond pump-probe transient absorption}

Transient absorption (TA) spectra were measured by a pumpprobe transient absorption spectroscopy system at room temperature $\left(25^{\circ} \mathrm{C}\right)$. The pump light is generated by a $\mathrm{Yb}: \mathrm{KGW}$ laser $(1028 \mathrm{~nm})$ operating at $5 \mathrm{~K} \mathrm{~Hz}$ with a pulse duration of 180 fs (PHAROS-SP-06-200, Light Conversion). Subsequently, the selected wavelength is obtained by an optical parametric amplifier (ORPHEUS-PO15F5HNP1, light conversion). The changes in absorption upon photoexcitation are probed with a white light continuum probe pulse $(450-900 \mathrm{~nm})$ that is generated by focusing a part of fundamental $1028 \mathrm{~nm}$ light in a sapphire crystal. TA data were acquired using a commercial TA spectrometer (HELIOS, Ultrafast Systems) by subtracting the probe signal with pump off from that with pump on $(\Delta A=$ $\left.\ln \left(I_{\mathrm{on}} / I_{\text {off }}\right)\right)$. To assure the stability of the pump pulse, the deviation of the pump power before and after each measurement was limited to $10 \%$. All compounds were measured in a quartz cuvette with $2 \mathrm{~mm}$ path length. All spectra were corrected for the chirp of the white probe light and the polarization effect due to the diffusive reorientation.

TA spectra of p-ADA and pp-ADA were analyzed by global analysis using the open source software Glotaran. ${ }^{32}$ In this method, the two-dimensional data were fitted by a sequential kinetic scheme with increasing lifetimes. This analysis yields a set of evolution-associated difference spectra (EADS), representing the spectral evolution of the excited species. The strength of the analysis can be assessed by the comparison between the original TA data and the fitted traces.

\section{DFT calculations}

All electronic structure calculations were performed using the Amsterdam Density Functional (ADF) software package (3,34 $^{33}$ with the CAM-B3LYP functional and the double-zeta plus polarization (DZP) basis set consisting of slater-type functions. The CAM-B3LYP functional was chosen since long-range corrected functionals are essential in accurately describing the energetics of excited states with substantial charge transfer character. ${ }^{35,36}$ Density functional theory (DFT) was used for the geometry optimization of the ground state molecules, in which both alkyl chains attached to the BDT unit were replaced by methyl groups. The optical absorption spectra were calculated by time-dependent density functional theory (TDDFT). ${ }^{37}$ Unrestricted DFT and TDDFT with a spin polarization of 1 were used to optimize the geometry and the excitation spectra of charged molecules, respectively. It should be noted that it has been shown that the 
two-electron contributions to the excited state that are not included in TDDFT will introduce an overestimate of the excitation energies for BODIPY, ${ }^{38,39}$ but this is prohibitive of the size of the molecules in this study.

To optimize the geometries of the excited states, the ground state gradients, together with the gradients of the TDDFT excitation energy were used to calculate the excited state gradients. ${ }^{40}$ To simulate the excited state formed upon photoexcitation at $500 \mathrm{~nm}$, the lowest vertical excited state from the ground state was optimized to obtain the relaxed geometry of the lowest excited state. The electronic structure of this relaxed excited state can then be compared to that of the vertical excited state corresponding to the ground state geometry. To allow the possibility of symmetry-breaking during the structural relaxation in the initial excited state, the excited state geometries of p-ADA and pp-ADA were optimized in both $C(\mathrm{i})$ symmetry and without symmetry. Furthermore, the geometry optimization of the charge transfer (CT) state was performed using the gradients of the second lowest allowed excitation that exhibits string CT character in the ground state geometry.

The delocalization of the relaxed excited state and the degree of the CT character were quantified by calculating the difference in charge distribution between the ground state and the excited state. For a given geometry, the charge distribution in the ground state was derived from the Mulliken charges on the donor, the acceptors and the phenyl units. The charge distribution in the excited state was summed over the different contributions to the excited state including single orbital transitions with a contribution $>1 \%$.

Finally, in order to elucidate the solvent stabilization effect in THF, the Conductor like Screening Model (COSMO) ${ }^{41}$ was used as the solvation method in state-specific single point calculations. The pure LE and CT state were calculated by specifying the corresponding electron occupation numbers based on the optimized geometries of relaxed LE state and CT state, respectively. Subsequently, the stabilization energy due to solvation for a specific state was determined by subtracting the solvent stabilization energy for its ground state from that for this specific state.

\section{Results and discussion}

We have studied the electronic structure/properties of three acceptor-donor-acceptor molecules consisting of one benzodithiophene as the electron donor and two BODIPY cores as the electron acceptors, connected by either one or two intervening phenyl units (Fig. 1). We have used a combination of steadystate absorption and emission spectroscopy, time-resolved transient absorption spectroscopy, and electronic structure calculations to characterize and understand the effects of D-A distance, geometric conformation (steric hindrance) and solvent polarity on the photophysics of these ADA triads.

\section{Steady-state optical properties}

A first indication of the electronic structure/properties can be obtained from steady-state absorption and emission spectra. a)

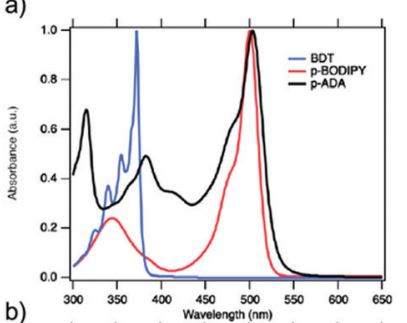

b)
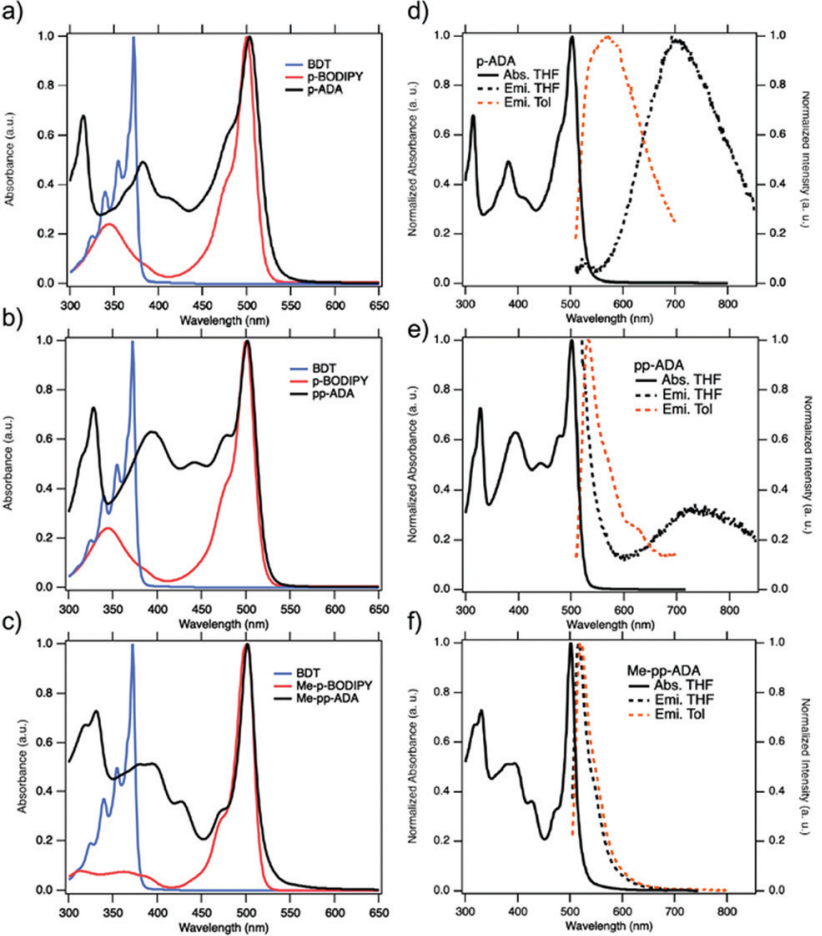

Fig. 2 Steady-state spectra. (a)-(c) Normalized absorption spectra of ADA triads and their model compounds in THF. (d)-(f) Normalized emission spectra of ADA triads in THF and toluene.

In Fig. 2a-c the UV/Vis absorbance spectra of all three compounds in THF are shown, together with those of the corresponding model donors and acceptors. All three compounds exhibit a dominant absorption peak at $\sim 500 \mathrm{~nm}$ originating from an $\mathrm{S}_{0}-\mathrm{S}_{1}$ transition on BODIPY. ${ }^{8}$ The much weaker $\mathrm{S}_{0}-\mathrm{S}_{2}$ transition on BODIPY below $400 \mathrm{~nm}$ is obscured by the strong absorption of BDT. The overlap between the spectra of ADA compounds and the acceptor units at $500 \mathrm{~nm}$ suggests that the electronic communication between the BODIPY acceptors and the BDT donor is very small in the ground state. Therefore, the photoexcitation at $500 \mathrm{~nm}$ is expected to result in a localized excited state on the BODIPY acceptor, generating a hole in the highest occupied fragment molecular orbital (HOFO) of BODIPY. On the other hand, compared to the absorption peak of the model BDT, a redshifted broad peak at $\sim 380-400 \mathrm{~nm}$ suggests a significant electronic interaction between BDT and the neighboring phenyl units. The steady-state emission spectra for the three ADA compounds are shown in Fig. 2d-f. In THF, an emission band with substantial Stokes shifts is observed in both p-ADA and pp-ADA, corresponding to the emission from the charge transfer (CT) state. In the non-polar toluene, the emission band of p-ADA is blue-shifted by more than $100 \mathrm{~nm}$. Noteworthy, with the longer donor-acceptor distance, the emission band of pp-ADA in toluene starts to resemble the characteristic emission of the model BODIPY acceptor more closely, indicating a more localized excited state on BODIPY acceptors. Finally, the sterically hindered Me-pp-ADA demonstrates a BODIPY-like emission band 
independent of the solvent polarity, indicating the absence of CT in this case.

\section{Time-resolved transient absorption spectra}

To study the dynamics of the excited state on an ultrafast timescale, the TA spectra of the ADA compounds in toluene and tetrahydrofuran (THF) were measured by femtosecond pump-probe spectroscopy. It is known that the charge transfer process in $\mathrm{D}-\mathrm{A}$ molecules is affected by the polarity of the environment. ${ }^{42-44}$ In a polar solvent, the highly dipolar CT state is stabilized by relaxation of the dipolar solvent, thereby favoring the charge transfer. In a non-polar solvent, the LE state is the dominant species upon excitation since the CT state is not stabilized by the surroundings. Hence, this solvation effect allows to study the absorption features of the LE state and CT state in a systematic way.

In toluene, a non-polar solvent, upon photoexcitation of p-ADA at $500 \mathrm{~nm}$ (Fig. 3a), a ground-state bleach (GSB) at $\sim 500 \mathrm{~nm}$ and an induced absorption band in the NIR (near-infrared) range appear simultaneously ( $<0.4 \mathrm{ps}$, orange curve). The GSB at $\sim 500 \mathrm{~nm}$ corresponds to the excited BODIPY acceptors. This GSB feature exhibits a fast initial decay immediately after the excitation pulse, which is accompanied by the further growth of the induced absorption band in the NIR as well as the formation of a small peak at $545 \mathrm{~nm}$. The broad featureless NIR absorption band between 600 and $900 \mathrm{~nm}$ reaches a maximum after $\sim 10 \mathrm{ps}$ and eventually decays concurrently with the decay of the GSB at longer times (Fig. S1, ESI $\dagger$ ). Interestingly, the NIR absorption band becomes less pronounced in pp-ADA (Fig. 3b) and completely disappears in Me-pp-ADApp-ADA (Fig. 3c). This shows that the steric hindrance introduced by the methyl groups decreases the electronic coupling with the
BODIPY moiety to such an extent that the photophysics completely change.

The absorption feature in the NIR regime can be found in other reported BODIPY derivatives with an analogous chemical structure, ${ }^{45,46}$ the origin of this absorption feature has hitherto escaped attention. In our ADA compounds, the formation of this absorption band should be associated with the ESA of the acceptor, because the charge transfer process is expected to be suppressed in the nonpolar solvent, and the energy transfer to the donor is energetically impossible. Furthermore, in comparison with the TA spectra of the model compound p-BODIPY or Me-pBODIPY (Fig. S2, ESI $\dagger$ ), where no induced absorption band was detected between $500 \mathrm{~nm}$ and $900 \mathrm{~nm}$, it is plausible to conclude that the ESA in P-ADA and Pp-ADA in the NIR regime originates from the ultrafast delocalization of the excited acceptors through the phenyl units onto the BDT donor to some extent, concurrently resulting in a delocalized excited state with partial charge transfer character. In Me-pp-ADA, this delocalization is completely hindered by the decoupling between the BODIPY core and the meso-phenyl unit, as evident by the absence of the absorption band in its TA spectrum.

The photophysics of p-ADA in toluene discussed above change quite drastically when going to the more polar solvent THF. Upon photoexcitation at $500 \mathrm{~nm}$, a similar ESA in the NIR regime is observed as in toluene but this feature decays in a few picoseconds, accompanied by the emergence of an absorption band with two maxima at $\sim 580 \mathrm{~nm}$ and $635 \mathrm{~nm}$ (Fig. 3d, grey dashed lines). This suggests the formation of a CT state stabilized by the solvent. The absorption feature at $580 \mathrm{~nm}$ is in accordance with the reported absorption spectra of radical anions of BODIPY analogues varying from 550-590 nm. ${ }^{11,47}$ Thus, the absorption feature at $635 \mathrm{~nm}$ is assigned to the BDT
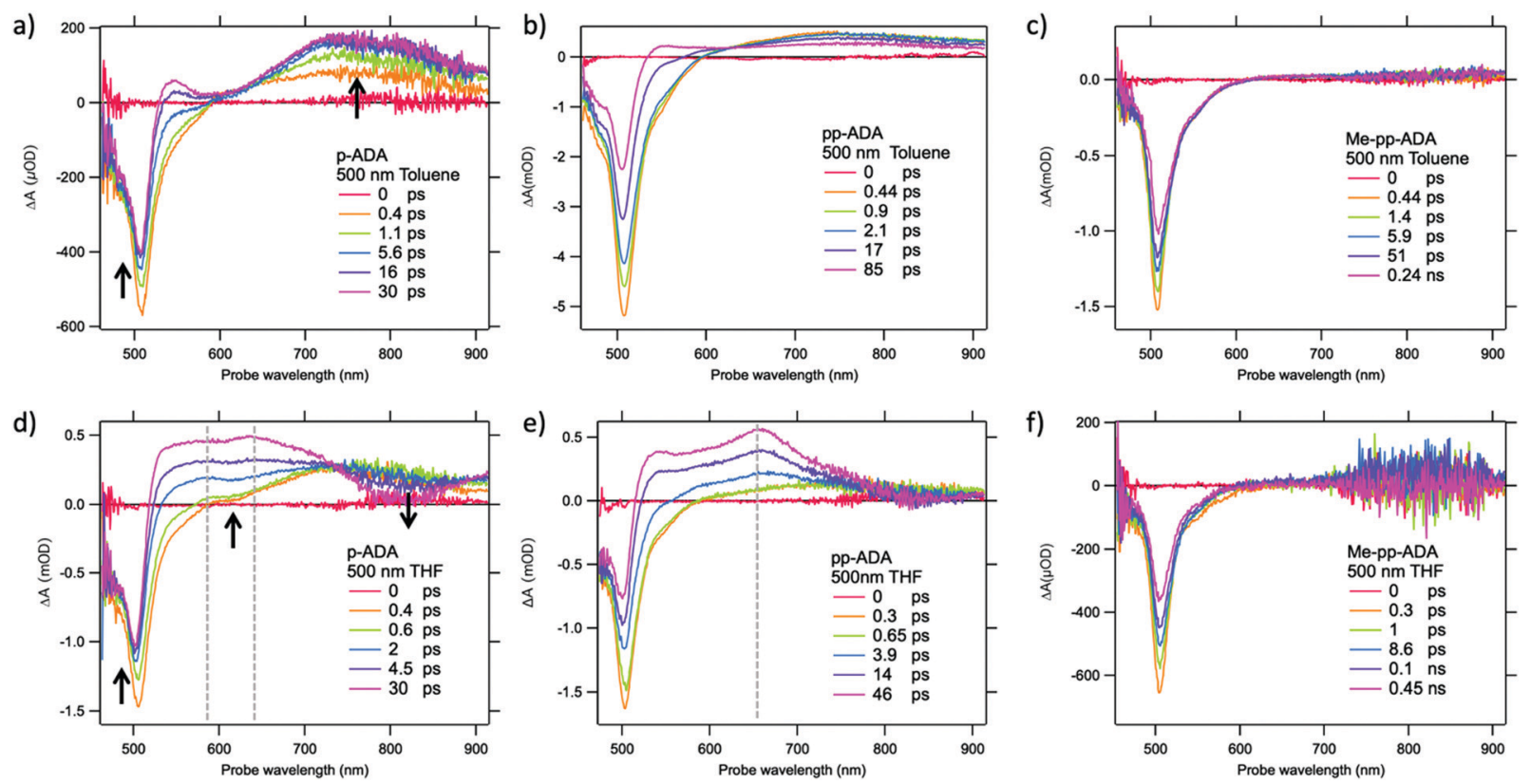

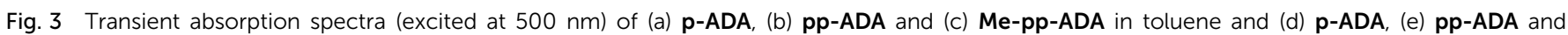
(f) Me-pp-ADA in THF. 
radical cation. Unfortunately, due to the novelty of incorporating BDT into D-A molecules, the absorption feature of an analogous BDT radical cation has not been reported, to our best knowledge.

Similar to the observation for p-ADA, in pp-ADA (Fig. 3e) a CT state is formed in THF with an absorption peak at $658 \mathrm{~nm}$ following the decay of the initial excited state. The red-shift in the absorption wavelength and the sharper absorption feature is likely associated with the red-shifted absorption of the BODIPY radical anions due to the increasing number of phenyl units, as supported by TDDFT calculations shown in Fig. S3 (ESI $\dagger$ ).

In the case of Me-pp-ADA (Fig. 3f), even in the polar solvent, no absorption of the delocalized excited state or the formation of the CT state was observed, implying that the excited state is strictly localized on the BODIPY acceptors. This is consistent with the perpendicular geometry obtained from DFT geometry optimizations, resulting in a zero electronic coupling between BODIPY and the rest of the molecule. The absence of any electronic coupling also blocks the charge transfer process. Moreover, DFT calculations also demonstrate that the adiabatic electron affinity for Me-p-BODIPY $(1.75 \mathrm{eV})$ is lower that for the model acceptor p-BODIPY (2.19 eV) (see Table S1, ESI $\dagger$ ). This considerable difference of 0.44 in the electron affinity shows that Me-p-BODIPY is a considerably weaker electron acceptor as compared with p-BODIPY. Therefore, the steric hindrance exerts a major impact on the photophysical properties of the ADA molecule through diminishing the electronic coupling across the molecule, which eventually precludes charge transfer in Me-pp-ADA.

The TA spectra of the ADA compounds photoexcited at 380 or $400 \mathrm{~nm}$ are presented in Fig. S4 and S5 (ESI $\dagger$ ). Generally, the TA spectra of p-ADA and pp-ADApp-ADA are similar to those obtained on excitation at $500 \mathrm{~nm}$, where the long-lived excited states are present in toluene, and the faster CT process is triggered in THF. Noticeable differences in the donor excitation arise from a slower formation of the GSB and a broader ESA band within 1 ps, indicating excitation energy transfer. However, it is nontrivial to disentangle the ESA of the donor from that of the acceptor and from coherent artifacts that are intrinsically present in the experiments to elucidate the excitation energy transfer on such an ultrafast timescale. In Me-pp-ADA, the excitation energy transfer is clearly evident in both toluene and THF as a result of the localized excited state on the acceptor. We have recently reported a more detailed analysis on the excitation energy transfer in Me-pp-ADA. ${ }^{31}$

\section{Global analysis}

To extract the quantitative information on the photophysical processes occurring on photoexcitation, the full kinetic matrices of p-ADA and pp-ADA at acceptor excitation were fitted by global analysis. We found that the evolution of the TA spectra is sufficiently described by a two-step kinetic scheme. Using global analysis, we obtained the evolution-associated difference spectra (EADS) consisting of the spectra of transient species and their lifetimes. The relevant photophysical processes are depicted in Fig. $4 \mathrm{c}$ and f. Detailed lifetimes are a)
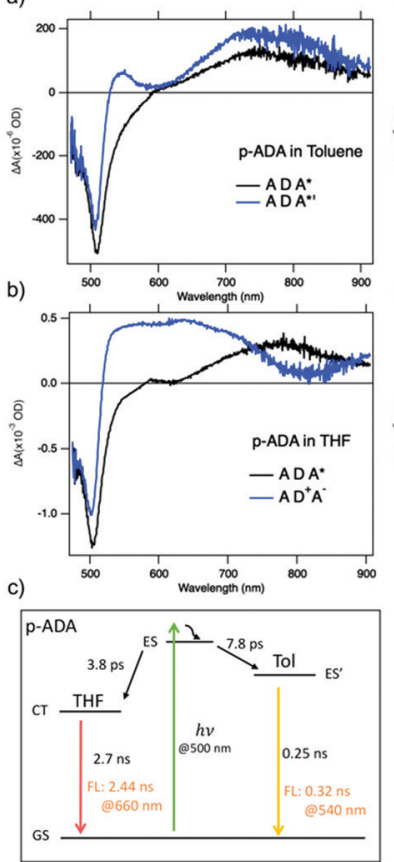

d)
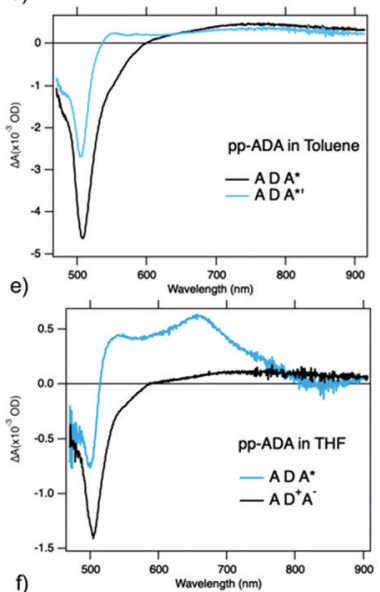

f)

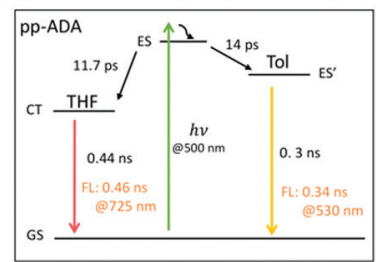

Fig. 4 Evolution-associated difference spectra of transient species of p-ADA in (a) toluene and (b) THF, and of pp-ADA in (d) toluene and (e) THF, and schematic illustration of relevant photophysical processes after photoexcitation at $500 \mathrm{~nm}$. Note that $\mathrm{ADA}^{\star \prime}$ and $\mathrm{ES}^{\prime}$ represent the delocalized excited state with the partial charge transfer character.

tabulated in Table S2 (ESI $\dagger$ ). The quality of the fitting can be justified by the consistency between the fitted traces and the original TA data (Fig. S6, ESI $\dagger$ ).

As presented in Fig. 4, the spectral signatures of EADS coincide with the photophysical processes discussed above. For p-ADA in both toluene and THF (Fig. 4a and b), photoexcitation initially leads to an excited state $\left(\mathrm{ADA}^{*}\right)$ with the same spectral features (black curve), independent of the solvent polarity. This initial (vertical) excited state is characterized by a GSB feature at $\sim 500 \mathrm{~nm}$ and a broad ESA in the NIR regime. The subsequent evolution of this excited state strongly depends on the solvent.

In toluene, with a representative time of $7.8 \mathrm{ps}$, a second species is formed with a lifetime of 0.25 ns (dark blue curve) showing a loss of amplitude in the GSB and a rise with the similar amplitude in the broad ESA. The preserved absorption feature in the NIR regime suggests a structural relaxation process in the excited state, whereas the small peak at $545 \mathrm{~nm}$ can be ascribed to the partial charge transfer character stemming from the relaxation-induced delocalization. Considering the similar florescence lifetime of $0.32 \mathrm{~ns}$ of the dominant species at $540 \mathrm{~nm}$ (Table S2 and Fig. S7, ESI $\dagger$ ) and a featureless fluorescence emission band, we assign this second species in toluene to be a delocalized excited state with the partial charge transfer character $\left(\mathrm{ADA}^{* \prime}\right)$. Hence, the initial excited state of $\mathbf{p - A D A}$ experiences an structural relaxation reaching a new electron density equilibrium in the presence of the solute-solvent interaction in toluene. ${ }^{48}$ 
In THF, the spectrum of the initial excited state (ADA*) is converted into the spectrum of the CT state $\left(\mathrm{AD}^{+} \mathrm{A}^{-}\right)$with a time constant of $3.8 \mathrm{ps}$. The CT state decays back to the ground state with a characteristic time of $2.68 \mathrm{~ns}$ as obtained from global analysis. This decay time closely matches the florescence lifetime of $2.44 \mathrm{~ns}$ at measured for the CT emission at $660 \mathrm{~nm}$ (Fig. S7, ESI $\dagger$ ).

In comparison with p-ADA, pp-ADA exhibits similar spectral characteristics and different rate constants for the charge transfer (11.65 ps) and charge recombination processes (0.44 ns). The slower CT rate upon lengthening the spacer is consistent with the attenuation of the rate expected for both the coherent tunneling and incoherent hopping mechanisms in D-A systems. ${ }^{49,50}$ The faster decay rate of the CT state in pp-ADA can be explained by the additional deactivation pathways induced by intramolecular rotations. ${ }^{26,45,51}$

\section{Molecular and electronic structure calculations}

In order to gain more insight into the nature of the initial (vertical) and relaxed excited states, we have performed electronic structure calculations. Geometry optimizations of the ground state (GS) and excited state (ES) were performed by TDDFT calculations using a CAM-B3LYP exchange correlation functional with a DZP basis set. Details about calculations are described in the Experimental section. The preliminary comparison between the excited p-BODIPY and Me-p-BODIPY pinpoints the limited conformational freedom in the ES posed by the steric hindrance (Fig. S8, ESI $\dagger$ ). Therefore, the ES geometry optimizations were focused on p-ADA and pp-ADA to study the effect of structural relaxation and different $\mathrm{D}-\mathrm{A}$ distances in the ES behaviors.

Given the optimized GS geometry (Fig. 5a), the major contributions to the lowest allowed vertical transition in p-ADA consist of transitions from occupied fragment molecular orbitals localized on the two BODIPY cores to the unoccupied orbitals on BODIPY cores (Table S3, ESI $\dagger$ ). As a result, the difference between the Mulliken charges in the lowest ES and GS is negligible as shown in Fig. 5b (black bar). This indicates a highly localized vertical ES. Subsequently, the gradients of the lowest excitation energy were used to optimize the geometry of the relaxed local excited (LE) state. When restricting the geometry to $C(\mathrm{i})$ symmetry, the ES optimization results in a decrease of $5.8^{\circ}$ in the torsion angles between the BODIPY core and the meso-phenyl (BODIPY-ph), accompanied by an increase of $4.5^{\circ}$ in the torsion angles between the BDT donor and the phenyl units. These geometry rearrangements result in a slightly increased delocalization in the ES (Fig. 5b, gray bar) and a lower $S_{1}$ energy (Fig. 5c). When symmetry breaking is allowed, in the ES geometry optimization a broken-symmetrystructure is obtained with an energy that is $0.054 \mathrm{eV}$ lower than for the symmetric ES. As shown in Fig. 5a, the symmetrybreaking structural relaxation in the ES yields a slightly reduced BODIPY-ph torsion angle of $51.9^{\circ}$ and an increased BODIPY-ph torsion angle of $61.9^{\circ}$. Consequently, the BODIPY-phenyl electronic coupling is increased on one side and decreased on the other side, splitting the energy levels of the LUMO and
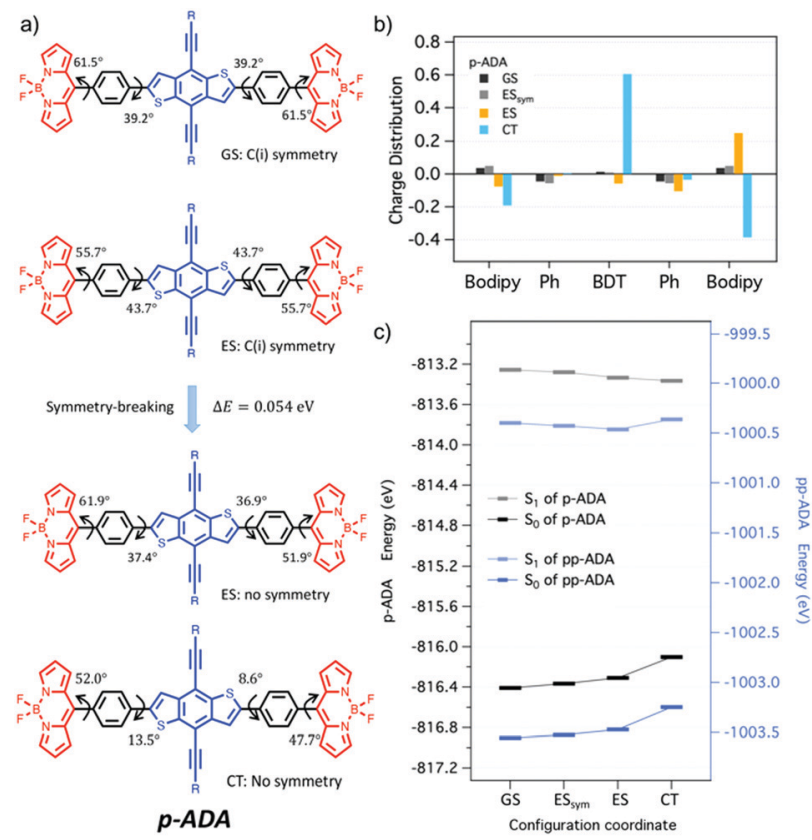

Fig. 5 Geometry optimization of the excited state. (a) Torsion angles in the geometry optimized ground state (GS), symmetrical excited state (ESsym), asymmetrical excited state (ES) and charge transfer state (CT) of p-ADA. (b) Geometry-dependent charge distribution of the excited state as compared to the ground-state following the lowest vertical transition of p-ADA. (c) Energy levels of $S_{0}$ and $S_{1}$ corresponding to the geometries of GS, ESsym, ES and CT of p-ADA and pp-ADA.

LUMO+1 that are degenerate in the symmetrical geometry. Along with the reduced BDT-ph torsion angles, the delocalization of the LE state is enhanced by the symmetry-breaking as evident in Fig. 5b (yellow bar) and Table S3 (ESI $\dagger$ ). Similar ES symmetry-breaking and its solvent dependence have been seen in other $\mathrm{ADA}$ or $\mathrm{DAD}$ triads using time-resolved infrared spectroscopy $^{52}$ or broadband fluorescence upconversion spectroscopy. 53

Finally, the geometry of the CT state in p-ADA was optimized using the excitation energy gradients of the second lowest allowed transition, which mainly consists of transitions from the occupied fragment molecular orbital on the BDT donor to the unoccupied fragment molecular orbital on the BODIPY acceptors with a weight of $54 \%$. Notably, the optimized geometry of the CT state is much more planar compared with that of the LE state (Fig. 5a). The concomitant delocalization of the electron density is manifested in its LUMO as shown in Table S3 (ESI $\dagger$ ). In this optimized CT geometry, the lowest vertical transition becomes CT-dominated, where the BDT-toBODIPY transition accounts for $78 \%$ of the total transition. Accordingly, the CT character prevails in the lowest transition leading to a large charge redistribution as quantified in Fig. $5 \mathrm{~b}$ (blue bar). Moreover, the lowest transition energy based on the CT geometry leads to a lower $\mathrm{S}_{1}$ energy than the local ES geometry in spite of its higher $S_{0}$ energy (Fig. 5c). This energetically favorable CT character further substantiates the delocalized excited state suggested by the comparison between the TA spectrum of p-ADA with that of the model compound p-BODIPY. 
Similarly, in pp-ADA the lowest allowed vertical transition corresponding to the GS geometry is mostly localized on BODIPY cores (Table S4, ESI $\dagger$ ). Driven by the symmetrybreaking geometry relaxation, the electron density becomes more delocalized in the $S_{1}$ as indicated by its charge distribution in Fig. S9 (ESI $\dagger$ ) (yellow bar). Compared with p-ADA, the longer D-A distance causes a smaller overall delocalization in the excited state in pp-ADA. The weaker delocalization in the excited pp-ADA indicated by the calculations is consistent with the much weaker induced NIR absorption in the TA spectra as well as the more BODIPY-like emission spectrum in toluene. Furthermore, the TDDFT geometry optimization following the second lowest allowed transition was used to obtain the CT geometry. However, based on this optimized geometry, $66 \%$ of the lowest transition is made up of the transition from the occupied fragment molecular orbital on the BDT to the unoccupied fragment molecular orbital on the BDT (Table S4, ESI $\dagger$ ). Hence, the degree of CT character in pp-ADA is smaller than that in p-ADA as shown in Fig. S9 (ESI $\dagger$ ). As a consequence, the $S_{1}$ energy associated with this CT geometry is less favorable than the $\mathrm{S}_{1}$ energy of the LE state (Fig. 5c). This attenuation in the CT character upon lengthening the $\mathrm{D}-\mathrm{A}$ distance is concurrent with the slower CT rate in pp-ADA indicated by the global analysis.

Considering that the solvating effect on the molecule in the experiments such as the stabilization is not taken into account by the calculations in the gas phase, the COSMO solvation method was used in state-specific DFT calculations to estimate the solvent stabilization for the pure LE state and the CT state. As listed in Table S5 (ESI $\dagger$ ), the CT state is generally stabilized more than the LE state in the polar solvent, as expected. Moreover, the more delocalized LE state in p-ADA shows a slightly larger solvent stabilization than that in pp-ADA. Although the solvent stabilization of the pure CT state is larger in pp-ADA, it is plausible that the overall smaller CT character in the excited pp-ADA smaller due to the longer D-A distance outweighs this larger solvent stabilization for the pure CT state.

\section{Conclusions}

In conclusion, we have examined the solvent-dependent ultrafast excited state dynamics of three acceptor-donor-acceptor triads by a combination of femtosecond transient absorption spectroscopy and complementary electronic structure (DFT/ TDDFT) calculations. In toluene, the photoexcitation yields a delocalized vertical excited state in p-ADA and pp-ADA. This excited state undergoes structural relaxation that gives rise to an excited state with partial charge transfer character. In THF, both red emissive p-ADA and pp-ADA triads show a fast charge transfer process triggered by the same initial excited state formed in toluene. Increasing the donor-acceptor distance leads to a slower charge transfer and a less pronounced delocalization in the excited state as shown by global analysis and TDDFT calculations, respectively. Noteworthy, manipulation of the molecular conformation through steric hindrance strongly affects the photophysical properties of such triads. Forcing the
BODIPY acceptor in a perpendicular conformation with respect to the conjugated spacer leads to a lowering of the electron affinity of the acceptor. In addition it disrupts electronic coupling across the molecule and therefore prohibits the formation of the delocalized excited state, which in turn suppresses the charge transfer process in Me-pp-ADA. These results show that by systematically varying the molecular structure, the balance between the population of the local excited state and the CT state can be tuned to a large extent. The improved understanding of the relation between molecular structure and the excited state processes will enable the design of the next generation of fluorescent molecular rotors based on BODIPY dyes for use in imaging and sensing applications.

\section{Conflicts of interest}

There are no conflicts to declare.

\section{References}

1 A. Athanasiadis, C. Fitzgerald, N. M. Davidson, C. Giorio, S. W. Botchway, A. D. Ward, M. Kalberer, F. D. Pope and M. K. Kuimova, Phys. Chem. Chem. Phys., 2016, 18, 30385-30393.

2 M. A. Haidekker, W. Akers, D. Lichlyter, T. P. Brady and E. A. Theodorakis, Sens. Lett., 2005, 3, 42-48.

3 I. Lopez-Duarte, T. T. Vu, M. A. Izquierdo, J. A. Bull and M. K. Kuimova, Chem. Commun., 2014, 50, 5282-5284.

4 M. K. Kuimova, G. Yahioglu, J. A. Levitt and K. Suhling, J. Am. Chem. Soc., 2008, 130, 6672-6673.

5 M. K. Kuimova, S. W. Botchway, A. W. Parker, M. Balaz, H. A. Collins, H. L. Anderson, K. Suhling and P. R. Ogilby, Nat. Chem., 2009, 1, 69-73.

6 M. A. Haidekker, A. G. Tsai, T. Brady, H. Y. Stevens, J. A. Frangos, E. Theodorakis and M. Intaglietta, Am. J. Physiol., 2002, 282, H1609-H1614.

7 Y. Y. Wu, W. T. Yu, T. C. Hou, T. K. Liu, C. L. Huang, I. C. Chen and K. T. Tan, Chem. Commun., 2014, 50, 11507-11510.

8 M. Kollmannsberger, K. Rurack, U. Resch-Genger and J. Daub, J. Phys. Chem. A, 1998, 102, 10211-10220.

9 K. Rurack, M. Kollmannsberger, U. Resch-Genger and J. Daub, J. Am. Chem. Soc., 2000, 122, 968-969.

10 Y. H. Cheng, J. G. Wang, Z. J. Qiu, X. Y. Zheng, N. L. C. Leung, J. W. Y. Lam and B. Z. Tang, Adv. Mater., 2017, 29, 1703900.

11 S. Hattori, K. Ohkubo, Y. Urano, H. Sunahara, T. Nagano, Y. Wada, N. V. Tkachenko, H. Lemmetyinen and S. Fukuzumi, J. Phys. Chem. B, 2005, 109, 19042.

12 E. J. McLaurin, L. R. Bradshaw and D. R. Gamelin, Chem. Mater., 2013, 25, 1283-1292.

13 D. F. Li, W. D. Hu, J. Wang, Q. W. Zhang, X. M. Cao, X. Ma and H. Tian, Chem. Sci., 2018, 9, 5709-5715.

14 H. F. Yao, L. Ye, H. Zhang, S. S. Li, S. Q. Zhang and J. H. Hou, Chem. Rev., 2016, 116, 7397-7457. 
15 L. Ye, S. Q. Zhang, L. J. Huo, M. J. Zhang and J. H. Hou, Acc. Chem. Res., 2014, 47, 1595-1603.

16 L. Ye, S. Q. Zhang, W. C. Zhao, H. F. Yao and J. H. Hou, Chem. Mater., 2014, 26, 3603-3605.

17 S. Sengupta, U. K. Pandey and E. U. Athresh, RSC Adv., 2016, 6, 73645-73649.

18 P. R. Aswathy, S. Sharma, N. P. Tripathi and S. Sengupta, Chem. - Eur. J., 2019, 25, 14870-14880.

19 H. Sunahara, Y. Urano, H. Kojima and T. Nagano, J. Am. Chem. Soc., 2007, 129, 5597-5604.

20 Y. Gabe, Y. Urano, K. Kikuchi, H. Kojima and T. Nagano, J. Am. Chem. Soc., 2004, 126, 3357-3367.

21 M. Kollmannsberger, K. Rurack, U. Resch-Genger and J. Daub, J. Phys. Chem. A, 1998, 102, 10211-10220.

22 G. Ulrich, R. Ziessel and A. Harriman, Angew. Chem., Int. Ed., 2008, 47, 1184-1201.

23 J. Karolin, L. B.-A. Johansson, L. Strandberg and T. Ny, J. Am. Chem. Soc., 1994, 116, 7801-7806.

24 T. G. Pavlopoulos, M. Shah and J. H. Boyer, Appl. Opt., 1998, 27, 4998-4999.

25 A. Loudet and K. Burgess, Chem. Rev., 2007, 107, 4891-4932.

26 R. R. Hu, E. Lager, A. Aguilar-Aguilar, J. Z. Liu, J. W. Y. Lam, H. H. Y. Sung, I. D. Williams, Y. C. Zhong, K. S. Wong, E. Pena-Cabrera and B. Z. Tang, J. Phys. Chem. C, 2009, 113, 15845-15853.

27 D. K. Zhang, V. Martin, I. Garcia-Moreno, A. Costela, M. E. Perez-Ojeda and Y. Xiao, Phys. Chem. Chem. Phys., 2011, 13, 13026-13033.

28 J. T. Buck, R. W. Wilson and T. Mani, J. Phys. Chem. Lett., 2019, 10, 3080-3086.

29 S. Toliautas, J. Dodonova, A. Žvirblis, I. Čiplys, A. Polita, A. Devižis, S. Tumkevičius, J. Šulskus and A. Vyšniauskas, Chem. - Eur. J., 2019, 25, 10342-10349.

30 S. Sengupta and U. K. Pandey, Org. Biomol. Chem., 2018, 16, 2033-2038.

31 S. Sharma, Z. Wei, F. Grozema and S. Sengupta, Phys. Chem. Chem. Phys., 2020, 22, 25514-25521.

32 J. J. Snellenburg, S. P. Laptenok, R. Seger, K. M. Mullen and I. H. M. van Stokkum, J. Stat. Softw., 2012, 49, 1-22.

33 G. t. Velde, F. M. Bickelhaupt, E. J. Baerends, C. F. Guerra, S. J. A. v. Gisbergen and J. G. S. a. T. Ziegler, J. Comput. Chem., 2001, 22, 931-967.

34 E. J. Baerends, D. E. Ellis and P. Ros, Chem. Phys., 1973, 2, 41-51.
35 A. Pedone, J. Chem. Theory Comput., 2013, 9, 4087-4096.

36 T. Yanai, D. P. Tew and N. C. Handy, Chem. Phys. Lett., 2004, 393, 51-57.

37 S. J. A. v. Gisbergen, J. G. Snijders and E. J. Baerends, Comput. Phys. Commun., 1999, 118, 119-138.

38 B. Le Guennic and D. Jacquemin, Acc. Chem. Res., 2015, 48, 530-537.

39 M. R. Momeni and A. Brown, J. Chem. Theory Comput., 2015, 11, 2619-2632.

40 M. Seth, G. Mazur and T. Ziegler, Theor. Chem. Acc., 2011, 129, 331-342.

41 A. Klamt and G. Schüürmann, J. Chem. Soc., Perkin Trans. 2, 1993, 799-805.

42 A. Weller, Z. Phys. Chem., 1982, 133, 93-98.

43 D. Inan, R. K. Dubey, W. F. Jager and F. C. Grozema, J. Phys. Chem. C, 2019, 123, 36-47.

44 H. N. Zhu, M. Li, J. P. Hu, X. Wang, J. L. Jie, Q. J. Guo, C. F. Chen and A. D. Xia, Sci. Rep., 2016, 6, 1-12.

45 T. Suhina, S. Amirjalayer, S. Woutersen, D. Bonn and A. M. Brouwer, Phys. Chem. Chem. Phys., 2017, 19, 19998-20007.

46 M. T. Whited, N. M. Patel, S. T. Roberts, K. Allen, P. I. Djurovich, S. E. Bradforth and M. E. Thompson, Chem. Commun., 2012, 48, 284-286.

47 B. Mangham, M. W. D. Hanson-Heine, E. S. Davies, A. Wriglesworth, M. W. George, W. Lewis, D. L. Kays, J. McMaster, N. A. Besley and N. R. Champness, Phys. Chem. Chem. Phys., 2020, 22, 4429-4438.

48 T. Kim, W. Kim, H. Mori, A. Osuka and D. Kim, J. Phys. Chem. C, 2018, 122, 19409-19415.

49 M. U. Winters, K. Pettersson, J. Martensson and B. Albinsson, Chem. - Eur. J., 2005, 11, 562-573.

50 H. Mcconnell, J. Chem. Phys., 1961, 35, 508-515.

51 H. L. Kee, C. Kirmaier, L. H. Yu, P. Thamyongkit, W. J. Youngblood, M. E. Calder, L. Ramos, B. C. Noll, D. F. Bocian, W. R. Scheidt, R. R. Birge, J. S. Lindsey and D. Holten, J. Phys. Chem. B, 2005, 109, 20433-20443.

52 B. Dereka, A. Rosspeintner, R. Stezycki, C. Ruckebusch, D. T. Gryko and E. Vauthey, J. Phys. Chem. Lett., 2017, 8, 6029-6034.

53 J. S. Beckwith, A. Rosspeintner, G. Licari, M. Lunzer, B. Holzer, J. Frohlich and E. Vauthey, J. Phys. Chem. Lett., 2017, 8, 5878-5883. 